\title{
CA IN SITU DEL CUELLO UTERINO* (Experiencia sobre 73 casos consecutivos)
}

TRABAJO PRESENTADO EN EL VI CONGRESO NACIONAL DE CIRUGIA - BOGOTA 1967

\author{
Dr. Alvaro Fonnegra Miramón** \\ Dr. Rubén Darío Guzmán Alandete*** \\ Dr. Armando Santamaría****
}

Este trabajo tiene por objeto continuar una revisión de casos de $\mathrm{Ca}$. In Situ diagnosticados, tratados $y$ controlados en el Servicio de Ginecología del Hospital San Juan de Dios de Bogotá, en un lapso comprendido entre Diciembre de 1962 y Junio de 1967. Los Doctores Fonnegra y Sarria, revisaron los casos ocurridos de Diciembre de 1962 a Junio de 1965, trabajo que fué presentado en el VI Congreso de Obstetricia y Ginecología verificado en la ciudad de Cartagena, en Diciembre de 1965. Hemos querido complementarlo aún más con estudios citológicos, gracias a la colaboración del Dpto. de Patología de nuestro Hospital.

Queremos recalcar la importancia de una buena historia y de un buen examen ginecológico complementado con especuloscopia, un estudio citológico sistemático y una toma de biopsia de cuello en caso de existir una lesión en dicha zona, previo test de Schiller. Tener en mente además, que a pesar de que encontremos un cuello aparentemente sano, no implica que podamos descartar un Carci- noma Intraepitelial. Estamos de acuerdo con Novak, quien opina que prácticamente ninguna mujer debiera morir de carcinoma si el médico aplicara estos principios; si pensara en esa forma y si la mujer se sometiera a revisiones periódicas en manos de un ginecólogo calificado y responsable, pues el Ca. In Situ diagnosticado precozmente da un margen de curación del $100 \%$ y al dejarlo evolucionar a un estado invasor, tomando en cuenta todos los estados, la curación es muy onerosa alcanzando apenas un $46 \%$; por eso es necesario que todas las mujeres sean examinadas por lo menos una vez al año, sobre todo al llegar a los 25 años y aún más si han tenido hijos.

* Trábajo elaborado en la Sección de Obstetricia y Ginecología del Hospital San Juan de Dios de Bogotá (Universidad Nacional) con la colaboración de la Sección de Patología (Universidad Nacional).

** Prof. Asoc. del Dpto. de Obst. y Gin. de la Universidad Nacional.

*** Prof. Asistente del Dpto. de Obst. y Gin. de la Universidad Nacional.

$\star * * *$ Prof. Asoc. del Dpto. de Patología de la Universidad Nacional. 


\section{MATERIAL Y METODO}

Se estudian 73 casos de Ca. In Situ diagnosticados, tratados y controlados de Diciembre de 1962 a Junio de 1967. Se incluye por lo tanto los 34 casos revisados por los doctores Fonnegra y Sarria. Se excluyen $14 \mathrm{ca}-$ sos, los cuales no pudieron ser estudiaclos debido a que no volvieron a la consulta de Ginecología, a pesar de nuestro interés por localizarlos. Creemos que con una mejor colaboración del Dpto. de Asistencia Social, podremos subsanar en parte este gravísimo problema. Actualmente estamos empeñados en esta campaña y así hemos podido incluir en este estudio varios casos que habíamos considerado perdidos por ausencia de la paciente.

\section{PARIDAD}

\begin{tabular}{llrr}
\hline Nulíparas & $=$ & 1 & $1,37 \%$ \\
Primíparas & $=$ & 5 & $6,85 \%$ \\
Multíparas & $=$ & 67 & $92.42 \%$ \\
& & - & $100.00 \%$ \\
\hline
\end{tabular}

En el cuadro anterior vemos que la mayor incidencia corresponde a las multíparas, lo cual está de acuerdo con lo encontrado por la mayoría de los autores. Concluyentes son los estudios de Gagnon, quien en una revisión que efectuó de historias y certificados de defunción de no menos 13.000 monjas canadienses no logró descubrir un solo caso de cáncer cervical.

Igualmente Pardo Vargas, encontró en su revisión, mayor número de carcinomas en las mujeres con mayor actividad sexual y en las que habían tenido mayor número de hijos.

\section{EDAD}

\begin{tabular}{llrr} 
Menos de 25 años & $=$ & 2 & $2,74 \%$ \\
Entre 26 y 35 años & $=$ & 21 & $28,77 \%$ \\
Entre 36 y 45 años & $=$ & 30 & $40,14 \%$ \\
Entre 46 y 55 años & $=$ & 14 & $19,18 \%$ \\
Más de 56 años & $=$ & 6 & $8,22 \%$ \\
\cline { 3 - 4 } & & &
\end{tabular}

Según Fluhmann, las edades más frecuentes oscilan entre 30 y los 45 años. Según otros autores la edad media oscila entre los 36 y los 41.6 años.

Mackay, Hertig y Younge, observaron un promedio de edad de 48 años para el Ca. Invasor, 38 años para el Ca. In Situ y 34.9 años, para la hiperplasia atípica. Los estudios de las series de Galvin y Telinde, mostró un índice de edades de 37.1 años; en la de Pund, de 36.6 años; en la de Younge, de 38 años. El hecho práctico es que existe una oportunidad que varía de meses a años, durante los cuales es posible diagnosticar el $\mathrm{Ca}$. Intraepitelial en su etapa curable en donde la citología vaginal desempeña un gran papel para estudiar mujeres asintomáticas con cuello aparentemente sano, antes de que evolucione a un $\mathrm{Ca}$. Invasor.

Vemos en el cuadro anterior que la mayor incidencia de Ca. In Situ, se encontró en mujeres entre los 36 a 45 años, lo cual está de acuerdo con lo encontrado por todos los autores. También notamos que son pocos los casos encontrados en las pacientes menores de 25 años, lo cual nos hace pensar que las campañas con el fin de descubrir el Carcinoma de Cuello uterino, debieron hacerse en mujeres mayores de 25 años. 
MOTIVO DE CONSULTA

\begin{tabular}{|c|c|c|c|}
\hline Dolor pélvico & $=$ & 12 & $16,44 \%$ \\
\hline Flujo genital & $=$ & 20 & $27,40 \%$ \\
\hline Hemorragia genital & $=$ & 17 & $23,29 \%$ \\
\hline Flujo y dolor pélvico & $=$ & 6 & $8,22 \%$ \\
\hline $\begin{array}{l}\text { Hemorragia genital y } \\
\text { dolor pélvico }\end{array}$ & $=$ & 3 & $4,11 \%$ \\
\hline $\begin{array}{l}\text { Sensación de cuerpo } \\
\text { extraño y/o inconti- } \\
\text { nencia urinaria de } \\
\text { esfuerzo }\end{array}$ & $=$ & 10 & $13,70 \%$ \\
\hline $\begin{array}{l}\text { Amenorrea } \\
\text { secundaria }\end{array}$ & $=$ & 2 & $2,74 \%$ \\
\hline Climaterio & $=$ & 1 & $1,37 \%$ \\
\hline \multirow[t]{2}{*}{$\begin{array}{l}\text { Vómito y dolor } \\
\text { retroesternal }\end{array}$} & $=$ & 1 & $1,37 \%$ \\
\hline & & 73 & $100.00 \%$ \\
\hline
\end{tabular}

El carcinoma intraepitelial básicamente es asintomático. En un estudio de Younge, Hertig y Armstrong señalaron que en $46 \%$ de 135 pacientes no tenían síntomas en absoluto, el $24 \%$ se quejaban de flujo genital y el $30 \%$ tenían hemorragias anormales. Hay que recordar dice Fluhmann, que el $\mathrm{Ca}$. Intraepitelial puede existir a pesar de la total ausencia de síntomas $y$ en un cuello aparentemente normal a simple vista y a la palpación.

Vemos que solamente 17 pacientes consultaron por hemorragia genital $(23,29 \%) 20$ consultaron por flujo genital $(27,40 \%)$ y 3 por hemorragia y dolor pélvico $(4,11 \%)$ yque otra buena parte consultaron por motivos no relacionados con el Carcinoma In Situ. Una paciente consultó por vómito y dolor retroesternal, presentaba un bocio, al practicarle un examen citológico rutinario, siendo positivo para malignidad nos hizo investigar por medio de una biopsia de cuello una pequeña ulceración que presentaba en esa zona.
PROLAPSOS

\begin{tabular}{llll} 
Cistorectocele II Sintomático $=$ & 6 & $8,22 \%$ \\
Prolapso genital de III grado $=$ & $\frac{4}{10}$ & $\frac{5,48 \%}{13,70 \%}$ \\
\hline
\end{tabular}

Se tiene el concepto que el carcinoma de cuello asociado a histerocele de III grado es raro, no existiendo una explicación satisfactoria, ya que es sorprendente que estando completamente exteriorizado el cuello tendrá más tendencia a sufrir traumatismos e irritaciones crónicas. Pardo Vargas, encontró en 31 casos de carcinoma de cuello estudiados, un caso de Ca. In Situ asociado con un Histerocele de III grado.

En nuestro estudio hallamos cuatro casos de Prolapso genital de III grado con $\mathrm{Ca}$. Intraepitelial, esto nos obliga a practicar biopsia de cuello a toda mujer que presente un histerocele de III grado con una ulceración por pequeña que ella sea.

\section{ESTADO DEL CUELLO UTERINO}

\begin{tabular}{llll}
\hline Cuello con erosión & $=50$ & $69,50 \%$ \\
Cuello aparentemente sano & $=23$ & $30,50 \%$ \\
& $\frac{73}{73}$ & $\frac{100,00 \%}{100}$ \\
\hline
\end{tabular}

Puede observarse un Ca. In Situ junto con una mucosa cervical de aspecto normal. No hay alteraciones macroscópicas que ayuden a identificar a la especuloscopia, un Ca. Intraepitelial; es más, algunos casos de cervicitis crónicas le dan un aspecto más anormal que un cuello con un Ca. Pre-invasivo, por lo tanto su aspecto macroscópico no desempeña un papel decisivo en el reconocimiento de esta enfermedad. El empleo rutinario de la citología exfoliativa es de suma importancia para descubrir esta entidad. 
Pardo Vargas, por medio de este procedimiento pudo descubrir siete casos de $\mathrm{Ca}$. In Situ en mujeres con cuellos sanos que no presentaban lesiones sospechosas.

Fluhmann en una serie de 48 enfermas encontró que $47,9 \%$ tenían un cuello normal y solamente el $18,7 \%$ mostraba cuellos uterinos que el Clínico consideró como "muy sospechosos".

En los casos presentados por nosotros, notamos que 23 de ellos (30,$50 \%$ ) presentaban cuellos aparentemente sanos y en los cuales la citología exfoliativa sistemática dio la voz de alarma para que hubiéramos investigado de una manera exhaustiva el cuello de esas mujeres.

BIOPSIA DE CUELLO

\begin{tabular}{|c|c|c|c|}
\hline $\begin{array}{l}\text { Positivas para Ca. In Situ } \\
\text { Cervicitis severa } \\
\text { Displasia y Cervicitis severa } \\
\text { Ca. Infiltrante } \\
\text { No se tomó biopsia }\end{array}$ & $\begin{array}{l}= \\
= \\
= \\
= \\
=\end{array}$ & $\begin{array}{r}59 \\
5 \\
7 \\
1 \\
1\end{array}$ & $\begin{array}{r}81,83 \% \\
6,85 \% \\
9,59 \% \\
1,37 \% \\
1,37 \%\end{array}$ \\
\hline & & 73 & $100,00 \%$ \\
\hline
\end{tabular}

A todas las pacientes excepto la última, se le tomó biopsia de cuello previa citología vaginal; se trataba de una paciente con un embarazo de más - menos 16 semanas, con dos estudios citológicos seguidos A5 y un cuello completamente sano.

Se pasó directamente a la conización, siendo positiva para Ca. Pre-invasivo.

El penúltimo caso, se trató de un error diagnóstico del patólogo. Fué valorado por nosotros como un estado 1 , habiéndosele practicado una histerectomía radical. Posteriormente el Dpto. de Patología al revisar los cortes anteriores de la biopsia y el útero extirpado, conceptuaron que se trataba de un Ca. In Situ y no de un infiltrante. En los demás casos el patólogo recomendó practicar conización pues presentaban citologías positivas.

\section{TRATAMIENTO DEFINITIVO}

\begin{tabular}{llll}
\hline $\begin{array}{l}\text { Conización } \\
\begin{array}{l}\text { Conziación más } \\
\text { histerectomía ampliada }\end{array}\end{array}$ & $=24$ & $32,88 \%$ \\
$\begin{array}{l}\text { Conización más histerectomía } \\
\text { ampliada más S.O.B. }\end{array}$ & $=$ & 13 & $17,81 \%$ \\
$\begin{array}{l}\text { Conización más histerec- } \\
\text { tomía vaginal }\end{array}$ & $=$ & 3 & $4,11 \%$ \\
$\begin{array}{l}\text { Histerectomía radical } \\
\text { Conización con extirpa- } \\
\text { ción del cuello restante } \\
\text { Histerectomía sin previa } \\
\text { conización }\end{array}$ & $=$ & 1 & $1,37 \%$ \\
& $=$ & 4 & $5,48 \%$ \\
\hline
\end{tabular}

\section{RESUMEN}

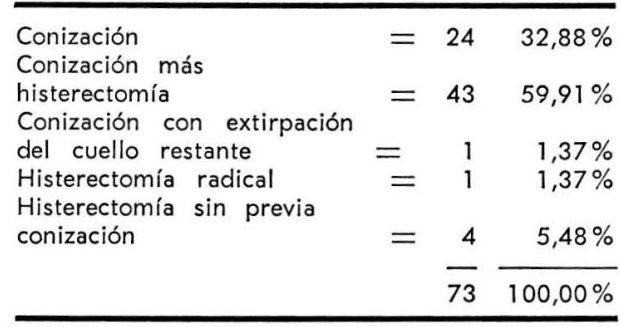

Como puede apreciarse, se practicaron 24 conizaciones como tratamiento definitivo $(32,88 \%)$ pues se trataba de mujeres jóvenes, excepto a una con un prolapso genital de III grado y mal estado general, a quien se consideró con gran riesgo quirúrgico para practicarle histerectomía vaginal. Estamos de acuerdo en aceptar esta conducta, como la sugiere Younge, Kottmeier y otros ginecólogos, en una mujer joven, deseosa de tener más hijos, si está dispuesta a someterse a controles ginecológicos estrictos a intervalos cortos, frotis y biopsias repetidas, sabiendo tanto ella como el marido cual es la situación. 
43 mujeres $(59,91 \%)$ fueron tratadas por medio de histerectomía abdominal o vaginal con o sin extirpación de ovarios, previa conización; a una se le practicó histerectomía radical por error del patólogo; a otra se le practicó conización y luego extirpación del cuello restante, pues ya había sido intervenida anteriormente de una histerectomía subtotal.

Exceptuando a la que se le practicó histerectomía radical, no se conizaron $4(5,48 \%)$ casos, procedimiento preliminar y necesario para confirmar el Ca. In Situ y descartar el Ca. Invasivo. El primero se trataba de una paciente con una enfermedad pélvica inflamatoria subaguda ya tratada lo cual hizo pensar que la conización podía reagudizarla; otra por dificultad técnica, pues la enferma presentaba un cuello muy alto y poco accesible durante el acto quirúrgico; los otros dos casos por error, conducta que no compartimos y que somos los primeros en criticar. En todos estos 4 casos se practicó histerectomía abdominal total ampliada.

De las 4 pacientes con prolapso genital de III grado, a 3 se les practicó histerectomía vaginal previa conización, excepto una por los motivos arriba anotados.

\section{CITOLOGIAS}

\begin{tabular}{llrr}
\hline A 5 & $=$ & 35 & $47,94 \%$ \\
A 4 & $=$ & 30 & $41,10 \%$ \\
A 3 & $=$ & 7 & $9,59 \%$ \\
A 2 & $=$ & $\frac{1}{73}$ & $\frac{1,37 \%}{100,00 \%}$ \\
\hline
\end{tabular}

Como puede apreciarse la mayor incidencia se presentó en las enfermas con citología A5 $(47,94 \%)$ y A4 $(41,10 \%)$ para un total de $89,04 \%$. Reagan y Hamonic, en 137 casos de $\mathrm{Ca}$. In Situ estudiados, encontraron que 120 casos $(87,85 \%)$, los frotis fueron positivos.

Encontramos un falso negativo, con una citología A2 $(1,37 \%)$. Se trataba de una paciente de 58 años que consultó por hemorragia genital; al examen ginecológico presentaba un pólipo endocervical; la biopsia de cuello reveló un $\mathrm{Ca}$. In Situ, posteriormente se le practicó una histerectomía abdominal ampliada previa conización.

Es digno de anotar la poca ocurrencia de falsos negativos lo cual se explica por la buena experiencia de nuestros Patólogos. Fluhmann sostiene que aproximadamente en un 10\% de los casos se obtienen citologías faltas negativas. Graham y Meigs, en un estudio de 40 casos de $\mathrm{Ca}$. In $\mathrm{Si}$ tu, encontraron 5 falsos negativos (12,5\%).

Todo esto nos pone de presente que sí se puede descubrir un $\mathrm{Ca}$ Preinvasivo a base de estudios citológicos, lo cual creemos que es el mejor procedimiento para llevar a cabo una campaña anticancerosa.

No debemos olvidar la importancia del estudio complementario por medio de la Colposcopia, pero desafortunadamente no se ha logrado rutinizar en el Servicio, habiéndose solamente practicado en 8 casos.

\section{PATOLOGIA QUIRURGICA ASOCIADA}

\begin{tabular}{llll}
$\begin{array}{lll}\text { Miomatosis uterina y quiste } \\
\text { paraovárico izquierdo }\end{array}$ & $=1$ & $1,37 \%$ \\
Adenomiosis & $=$ & $2,74 \%$ \\
Mioma pediculado & $=1$ & $1,37 \%$ \\
endocervical & $=$ & 1 & $1,37 \%$ \\
Divertículo de Meckel & & \\
& & \\
\hline
\end{tabular}

La salpinguectomía uni o bilateral estuvo condicionada a la patología encontrada en la trompa o al criterio del cirujano. 


\section{COMPLICACIONES POST-OPERATORIAS} DE LA CONIZACION

\begin{tabular}{llll}
\hline Hemorragia abundante & $=$ & 8 & $10,96 \%$ \\
Hematometra & $=$ & 1 & $1,37 \%$ \\
\cline { 2 - 2 } & & $\frac{9}{12,33 \%}$ \\
\hline
\end{tabular}

De los 8 casos de hemorragia abundante, 7 cedieron con taponamiento vaginal a gran presión y el otro al extirpar el cuello restante, detalle pasado por alto en el examen de ingreso, pues la paciente había sido sometida a una histerectomía subtotal.

Es bueno anotar que 2 pacientes se conizaron estando embarazadas no presentando complicación alguna.

El caso de la hematometra se trató con una dilatación instrumental del cuello. Darnalt R. presenta 87 casos de conización y 15 de ellos presentaron hemorragia abundante y 2 casos presentaron estenosis cervical.

\section{COMPLICACIONES POST-OPERATORIAS DE LA HISTERECTOMIA ABDOMINAL AMPLIADA}

$\begin{array}{llll}\text { Bronquitis y evisceración } & = & 1 & 1,37 \% \\ \text { Absceso de cúpula vaginal } & = & 4 & 5,48 \% \\ \text { Absceso de pared } & = & 5 & 6,85 \% \\ & & 10 & 13,70 \%\end{array}$

Todas estas complicaciones cedieron con un tratamiento adecuado a base de antibióticos y drenajes de los abscesos, según el caso.

De la mayor importancia es la corelación entre biopsias y piezas quirúrgicas extirpadas. En el análisis del cuadro anterior observamos que en 52 casos $(72,24 \%)$ la Biopsia para Ca. In Situ coincidió con la conización o histerectomía, según la indicación del caso.

A dos de estas pacientes se les practicó histerectomía, sin pasar por la fase de conización, una por error de conducta, que somos los primeros en criticar; y la otra por trastarse de una enfermedad pélvica subaguda.

Otra paciente con $\mathrm{Ca}$. In Situ en la biopsia, resultó con un un $\mathrm{Ca}$. In Situ micro-invasivo en la histerectomía abdominal ampliada; tampoco se practicó conización previa, por error.

En 7 pacientes con biopsia positiva para $\mathrm{Ca}$. In Situ, resultaron con diagnóstico final de displasia severa y/o cervicitis $(9.59 \%)$. Le atribuímos lo anterior, posiblemente a que en la biopsia el carcinoma muy incipiente, fué removido en su totalidad.

Al revés de lo anterior en 12 enfermas con citologías repetidas $A 3$, $\mathrm{A} 4$, ó A5 biopsias negativas para $\mathrm{Ca}$. In Situ, pero positivas para displasias severas $\mathrm{y} / 0$ cervicitis severas, resultaron a la postre 10 con un $\mathrm{Ca}$. In Situ y 2 asociado con un $\mathrm{Ca}$. escamo-celular micro-invasivo (16,44\%). Kayser y cols. al estudiar 608.000 exámenes citológicos encontraron un índice de presencia de la combinación de las 2 variedades de cánceres ( $\mathrm{Ca}$. In Situ + Ca. escamo-celular invasor) de 0,40\%.

De estas 12 pacientes comentamos 2 casos:

Una paciente de 62 años de edad, prolapso genital de III grado, biopsia positiva para cervicitis crónica, citologías $A 3$ y $A 4$, se le practicó conización; el resultado de la A.P. fué de un $\mathrm{Ca}$. In Situ con invasión glandular $+\mathrm{Ca}$. escamo-celular, micro-invasivo. Se remitió al I. N.C. para su tratamiento complementario; en esa Institución le practicaron operación de Schauta, en cuyo útero no se encontró tumor residual.

Otra con diagnóstico de displasia severa + cervicitis crónica severa, se le practicó histerectomía ampliada con S.O.B. sin pasar por la 
CORRELACION ENTRE BIOPSIAS Y PIEZAS QUIRURGICAS

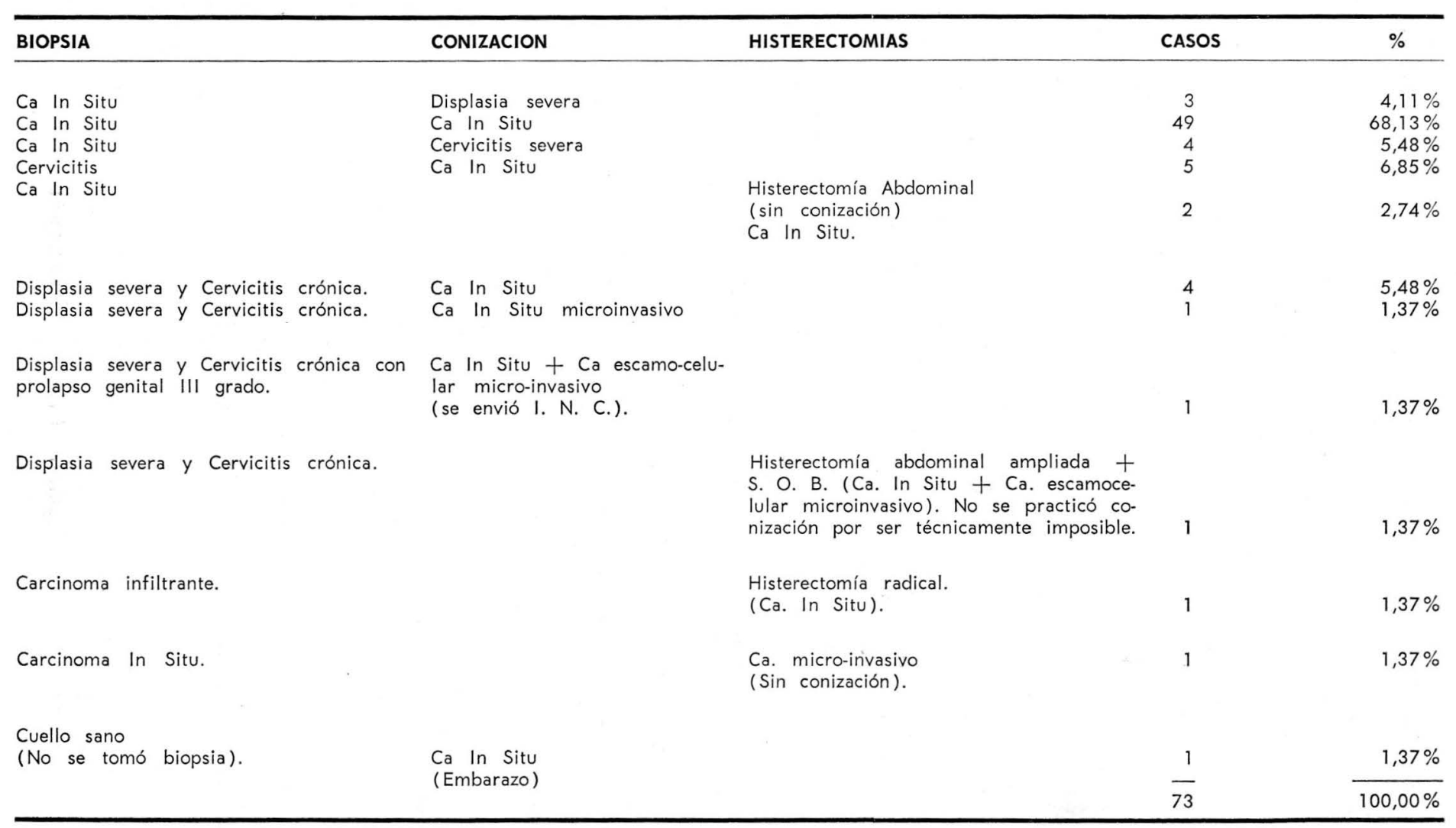


fase de conización, por ser técnicamente imposible; el diagnóstico final reveló un Ca. In Situ + Ca. escamo-celular micro-invasivo. Consultado al Patólogo, se consideró que por ser tan mínima la invasión, no se justificaba ningún otro tratamiento, sino control periódico riguroso. En consecuencia toda displasia asociada o no a citologías sospechosas o sugestivas de malignidad debe ser exhaustivamente estudiada.

Un caso con biopsia positiva para carcinoma infiltrante de cuello, se le practicó histerectomía radical; la pieza quirúrgica demostró un $\mathrm{Ca}$. In Situ.

Por último, en una embarazada con cuello sano y en la cual no se tomó biopsia previa, pero por citología A5 se conizó, resultó con un Ca. In Situ.

Tenemos que explicar que en nuestro servicio no practicamos sino una serie de biopsias y pasamos directamente a la fase de conización o histerectomía radical según el caso. No le vemos objeto ejecutar segunda o tercera series de biopsias por considerarlas superfluas y demorar el caso para su diagnóstico más de lo necesario.

TUMOR RESIDUAL

TUMOR RESIDUAL IN-UTERO EN LA HISTERECTOMIA

2 casos

$2,74 \%$

TUMOR RESIDUAL POST-CONIZACION

1 caso

$1,37 \%$

En la serie estudiada por nosotros solamente encontramos tres casos de Ca. In Situ residual, dos post-histerectomía abdominal ampliada (2,$74 \%)$, y otro postconización (1,$37 \%)$.
El primer caso se trataba de una paciente de 35 años de edad con citología A5, biopsia de cuello positiva para Ca. In Situ; en la conización se volvió a encontrar el Ca. In Situ. Como tratamiento definitivo se le practicó una histerectomía abdominal ampliada, encontrándose nuevamente el tumor In Situ residual.

El segundo caso similar al anterior en una enferma con edad superior a los 40 años.

El tercero se trataba de una paciente de 36 años de edad, dos citologías A5, biopsia de cuello positiva para Ca. In Situ con invasión glandular temprana y un embarazo de nıás - menos 16 semanás. Se le practıcó conización encontrándose un $\mathrm{Ca}$. In Situ en el corte de sección del cono. Siguió siendo sometida a controles clínicos y citológicos rigurosos durante su embarazo; la primera citología al mes de la conización demostró A3 y la última efectuada dos meses después reveló una citología A1. Se pensó dejar evolucionar su embarazo hasta el término, parto por vías naturales, y posteriormente en el puerperio volver a reconsiderar el caso con el objeto de practicar o no una histerectomía abdominal ampliada, con previa reconización, pero el control citológico próximo al finalizar su embarazo mostró un A5, con cuello sano.

La conducta en este difícil y suigéneris caso, fué ampliamente debatida en reunión de Profesores del Servicio, acordándose practicar una cesárea extemporánea seguida en el mismo acto quirúrgico de histerectomía abdominal ampliada. En la pieza quirúrgica de lútero extirpado se encontró Ca. In Situ en el cuello uterino residual.

Algunos autores norteamericanos dan cifras muy altas para tumor re- 
sidual post-conización; así Younge presenta el 17\%; Arvan y Davis el $21 \%$ y Boyd el $10 \%$. Nosotros, como se puede apreciar en el cuadro correspondiente solamente tuvimos un caso $(1,37 \%)$, y dos casos de tumor residual post-histerectomía ampliada $(2,74 \%)$. Darnalt sobre 22 casos de conización informa de dos casos con tumor residual In Situ y otro de $\mathrm{Ca}$. escamo-celular micro-invasivo, para un $13,85 \%$.

\section{CONTROLES}

\begin{tabular}{lllrr}
\hline Más de 6 meses & $=$ & 18 & $24,66 \%$ \\
Más de 12 meses & $=$ & 22 & $30,14 \%$ \\
Más de 24 meses & $=$ & 15 & $20,55 \%$ \\
Más de 36 meses & $=$ & 4 & $5,48 \%$ \\
No volvieron a control & $=$ & 14 & $19,18 \%$ \\
\cline { 2 - 2 } & & $\overline{73}$ & $100,00 \%$
\end{tabular}

Si hay una cosa importante en Cancerología es el control de las pacientes. Solamente así se puede evaluar la bondad de los tratamientos efectuados y hablar de curación del cáncer.

Afortunadamente en nuestro medio hemos logrado reunir un buen porcentaje de pacientes controladas, gracias a los servicios de Asistencia Social del Hospital San Juan de Dios de Bogotá, que se encarga de localizar y traer las enfermas al servicio de Clínica de Tumores que tenemos establecido, así hemos conseguido controlar el $79.2 \%$ de las pacientes tratadas para carcinoma cifra que la consideramos excelente para nuestro medio.

Es de anotar que dentro de las que no volvieron a control hay 6 casos que solo llevan entre 3 y 6 meses de tratamiento.

Solamente tuvimos un caso de reproducción del carcinoma al año y medio de tratamiento, después de conización al parecer insuficiente y co- mo tratamiento definitivo, que desarrolló un carcinoma infiltrante. Irradiada recientemente se encuentra en buenas condiciones generales en la actualidad.

La norma de control que seguimos en el Servicio de Ginecología es la siguiente: durante el primer año se efectúan controles periódicos cada tres meses y posteriormente cada 6 meses.

\section{RESUMEN Y CONCLUSIONES}

1) Se hace un estudio de 73 casos de Carcinoma In Situ, diagnosticados, tratados y controlados en el Servicio de Ginecología del Tospital San Juan de Dios de Bogotá, en un lapso comprendido de Diciembre de 1962 a Junio de 1967. Se excluyen 14 casos los cuales no volvieron a la consulta de ginecología.

2) La mayor incidencia correspondió a las pacientes multíparas, lo cual está de acuerdo con lo encontrado por la mayoría de los autores.

3 ) El mayor porcentaje (40,14\%), correspondió a aquellas entre los 36 y los 45 años, lo cual también está de acuerdo con los hallazgos de otros autores. El hecho práctico es que existe una oportunidad que varía de meses a años, durante los cuales es posible diagnosticar el $\mathrm{Ca}$. Intraepitelial en su etapa curable, en donde la citología exfoliativa desempeña un gran papel para estudiar mujeres asintomáticas con cuello aparentemente sano, antes de que evolucione a un Ca. Invasor.

El escaso porcentaje de casos encontrados en mujeres menores de 25 años $(2,74 \%)$, nos hace pensar que las campañas con el fin de hacer la pesquisa del Carcinoma In Situ debieran establecerse en mujeres mayores de 25 años. 
4) El Ca. Intraepitelial básicamente es asintomático. En nuestro estudio solamente 17 mujeres (23,29\%), consultaron por hemorragia genital; 20 consultaron por fuljo genital (27,$40 \%$ ), y 3 por hemorragia y dolor pélvico $(4,11 \%)$, y otra buena parte consultaron por motivos no relacionados con el carcinoma pre-invasivo.

5) Se encontraron 4 casos (5,$48 \%$ ), en donde existía la asociación de Histerocele de III grado con Ca. In Situ, esto nos obliga a practicar biopsia de cuello a toda mujer que presente histerocele de III grado con una ulceración del cuello por pequeña que ella sea.

6) Encontramos que 23 mujeres $(30,50 \%)$, tenían al examen ginecológico con espéculo un cuello aparentemente sano, por lo tanto su aspecto no desempeña un papel decisivo en el reconocimiento de esta enfermedad. Consideramos la citología vaginal rutinaria como el método ideal para descubrir el Ca. Intraepitelial en mujeres con cuello sano o con lesiones mínimas del mismo.

7) De los 73 casos estudiados, la biopsia fué positiva para $\mathrm{Ca}$. In Situ en $59(81,83 \%)$. En los casos restantes, excepto en uno en donde no se tomó biopsia de cuello el patólogo recomendó practicar conización pues presentaban citologías positivas.

8) Como tratamiento definitivo a 24 mujeres $(32,88 \%)$, se les practicó conización pues se trataba de mujeres jóvenes con deseos de tener hijos y fáciles de controlar. La gran mayoría $(59,91 \%)$, se les practicó histerectomía abdominal ampliada o vaginal con o sin extirpación de anexos, tratamiento que creemos es el más adecuado para tratar estas pacientes. A cuatro enfermas (5,48\%), no se les practicó conización previa; una por ser técnicamente imposible y otra porque presentaba una enfermedad pélvica inflamatoria sub-aguda ya tratada, lo cual hizo pensar que la conización podría reagudizarla; las otras dos restantes por error.

9) La mayor incidencia se presentó en las enfermas con citología A5 $(47,94 \%)$, A4 $(41,10 \%)$ y luego con citología A3 $(9,59 \%)$ esto nos pone de presente que sí se puede un $\mathrm{Ca}$. Pre-invasivo descubrir a base de estudios citológicos, lo cual consideramos el mejor procedimiento para llevar a cabo una campaña anti-cancerosa. Es digno de anotar la poca ocurrencia de "falsos negativos".

10) Solamente encontramos 5 casos $(6,85 \%)$, de patología quirúrgica asociada, los cuales se trataron según el caso.

$11)$ Muy pocos fueron los casos de complicaciones post-operatorias de la conización $(12,33 \%)$, y de la histerectomía abdominal ampliada (13,$70 \%$ ), los cuales cedieron con un tratamiento adecuado.

12) En 52 casos $(72,24 \%)$, el Ca. In Situ de la biopsia coincidió con la conización o histerectomía; en 7 casos $(9,59 \%)$, la biopsia positiva para $\mathrm{Ca}$. In Situ no coincidió con la conización, lo cual explic aque el carcinoma estaba muy localizado, fué removido en su totalidad; en 12 casos de displasias severas $\mathrm{y} / 0$ cervicitis severas, con citología A3, A4 ○ A5, resultaron finalmente con un $\mathrm{Ca}$. In Situ 10 y 2 asociado con un $\mathrm{Ca}$. escamocelular microinvasivo. Por consiguiente toda displasia con citología sospechosa o sugestiva de malignidad debe ser investigada rigurosamente.

13) En el servicio de Ginecología del Hospital San Juan de Dios no se practica sino una serie de biopsias, 
por considerar superfluas e innecesarias la segunda 0 tercera serie.

14) Se encontraron 3 casos de $\mathrm{Ca}$. In Situ residual; dos post-histerectomía abdominal ampliada $(2,4 \%)$ y otro post-conización (1,37\%). Esto contrasta con las cifras dadas por algunos autores norteamericanos y nacionales, quienes encuentran porcentajes mucho más altos.

15) Se logró obtener un porcentaje de controles del 79,2\%; cifra que consideramos excelente para nuestro medio.

\section{BIBLIOGRAFIA}

1) FONNEGRA M. ALVARO y SARRIA OLCOS CARLOS. Estado actual del carcinoma In Situ del cuello uterino, en el Hospital San Juan de Dios de Bogotá, revista Col. de Obstetricia y Ginecología. Vol. XVI, Noviembre y Diciembre de 1965. Pág. 430 y ss.

2) ANGEL GILBERTO. Estado actual del carcinoma del Cuello Uterino. Rev. Col. Obs. y Gin. Vol. GV No 133:43 1954.

3) CARDONA PEDRO N. Diagnóstico precoz del Cáncer por medio de la citología. Rev. Col. de Obs. y Gin. C. Vol. X № 5327: 338. 1959.

4) DARNALT E. JORDAN G. Ca. Intraepitelial. Memorias del II Congreso de Obs. y Gin. Cali. Colombia.

5) ISAZA MEJIA G. RODRIGUEZ RODRIGUEZ L. Informe estadístico sobre cincuenta mil ochocientas sesenta y seis (50.886) citologías del ICSS. Rev. Col. Obs. y Gin. Vol. XVI. Nov.-Dic. 1965. Pág. 446 y ss.

6) DARNALT RESTREPO E. Conización del cuello uterino. Rev. Col. Obs. y Gin. Vol. XVI. Nov.-Dic. 1965. Pág. 452 y ss.

7) LOPEZ E. GUILLERMO. Lesiones distróficas y Ca. In Situ del cérvix. Rev. Col. Obs. y Gin. Vol. VIII № 5. 1957.

8) LOPEZ E. GUILLERMO. Carcinoma intraepitelial del cérvix uterino. Rev. Col. Obs. y Gin. Vol. IX No 4. $333: 360.1958$.

9) LOPEZ E. GUILLERMO. Diagnóstico precoz del cáncer (Citología y cáncer extraepitelial. Repertorio de medicina y cirugía. Hospital San Juan de Dios. Vol. 4 № 5. $291: 296.1959$.
10) POSADA MARIO, VASQUEZ G. Citología exfoliativa del carcinoma del cuello uterino. Rev. Col. Obst. y Gin. Vol. 11 № 4. 376 380. 1960.

11) DIPAOLA GUILLERMO. Pesquisas de los estados iniciales del carcinoma cervical. Rev. Col. Obst. Gin. Vol. 14 № 1 13:20 1963.

12) NOVAK E. JONES G. JONES HOWARD. Tratado de Ginecología, Editorial Interamericana. 7: Edición 1966.

13) TELINDE W. RICHARD. Ginecología Operatoria. Editorial José Bernades. 2a Edición. 1958.

14) FLUHMANN FREDERIC C. El cuello uterino $y$ sus enfermedades. Editorial Salvat. 1: Edición 1963.

15) BENSON C. RALPH. Manual de Gin. y Obst. Editorial El Manual Moderno. 1? Edición 1966.

16) YOUNGE P. A. Premalignant lesions of the cervix and clinical management. Clin. Obst. y Gyn. 5. 1.137 sig. 1962.

17) YOUNGE P. A. Cancer of the uterine cervix. A. Preventable disease Obst. \& Gyn. Vol. 10469 sig. 1957.

18) PARDO VARGAS FCO. Cáncer del cuello uterino. Rev. Col. de Obst. y Gin. Vol. XVII Marzo abril de 1966.

19) KOTTMAIER H. L.: Current Treatment of carcinoma of the cervix. Amer. J. Obstet Gynec. 76: 243. 1958.

20) HERTIG, A. T. Y YOUNGE P. A.: What is cancer in situ of the Cervix? Is it the preinvasive form of true carcinoma? Amer. J. Obst. Gyn. 64:807, 1952.

21) YOUNGE, P. A. HERTIG, A. T. ARMSTRONG. D.: A study of 135 cases of carcinoma In Situ of cervix at Lree Hospital for Women. Amer. J. Obst. Gynec. 58: 867. 1949.

22) ENTERLINE E. T., ARVAN D. A. y DAVIS, R. E.: The predictability of residual carcinoma In Situ from study of cervical cones. Amer. J. Obstet and Gyn. 85: 940, 1963.

23) BOYD, J. R. y cols. Conservativ management of in Situ carcinoma of the cervix. Amer. J. Obst. Gyn. 85: 322, 1963.

24) ROVINSKY JOSEPH J. GUTTMACHER ALAN F. Complicaciones médicas, quirúrgicas y ginecológicas en el embarazo. Edit. Interamericana, S. A. 2a Edición. Pág. 298.

25) KAYSER y cols. Uterine citology pub. Health Rep. 75: 423-60.

26) REAGAN J. W. Y HAMONIC M. J.: Cancer. 385. 1956. 